ACEs: Evidence, Gaps, Evaluation and Future Priorities

\title{
ACEs: Evidence, Gaps, Evaluation and Future Priorities
}

\author{
Andrew Steptoe*, Theresa Marteau $* *$, Peter Fonagy $* * *$ and Kathryn Abel ${ }^{\dagger}$
}

*Department of Behavioural Science and Health, University College London.

E-mail: a.steptoe@ucl.ac.uk

**Department of Public Health and Primary Care, University of Cambridge.

E-mail:Tm388@cam.ac.uk

***Division of Psychology and Language Sciences, University College London.

E-mail: p.fonagy@ucl.ac.uk

${ }^{\dagger}$ Division of Psychology and Mental Health, University of Manchester.

E-mail: Kathryn.M.Abel@ manchester.ac.uk

\begin{abstract}
There is strong evidence linking adverse childhood experiences (ACEs) and poor outcomes in adulthood both in terms of mental and physical health. Gaps in both the evidence base and research priorities still exist. These include understanding how to identify and assess risk in children who have experienced ACEs, and also the development and, importantly, the evaluation of interventions. Outstanding gaps include whether there are sensitive periods during childhood, the role of resilience/protective factors, the causal relationships, biological mechanisms and relative risk of ACEs for particular negative outcomes. ACEs affect individual children differently and chronic exposure appears to increase the risk of poor outcomes in adulthood, meaning interventions should also be tailored to the individual children, families and communities. Generally, there needs to be better evaluation of interventions and dissemination of this information to ensure that their use is evidence based. More input from affected communities, clinicians, funding bodies and Government
\end{abstract}


ACEs: Evidence, Gaps, Evaluation and Future Priorities

departments is required to identify research priorities and ensure gaps in the evidence base are addressed.

Keywords: ACEs, children, early intervention, evidence-base, outcomes.

\section{Introduction}

The past few decades has seen increasing international interest in how experiences during childhood and adolescence, such as physical, sexual and emotional abuse and exposure to domestic violence, abuse of drugs and parental mental illness, can affect long term health. Increasingly evidence links such adverse childhood experiences (referred to as ACEs) with poor mental and physical health outcomes in adulthood. This article focuses on the evidence base and existing gaps linking ACEs with poor health outcomes and considers evidence for interventions. It is based on a submission by the Academy of Medical Sciences to the UK House of Commons Science and Technology Committee Inquiry into evidence-based early years intervention: http://data.parliament.uk/writtenevidence/committeeevidence.svc/ evidencedocument/science-and-technologycommittee/evidencebased-early-yearsintervention/written/75209.html

\section{Evidence-base for the link between ACEs and long-term negative outcomes}

ACEs can be broad in nature, and include mental/physical/sexual abuse, neglect, parental dysfunction/mental illness, or parental loss. There is strong evidence linking ACEs with longterm negative outcomes in areas such as mental health (Lereya et al., 2013; Geoffroy et al., 2014; Mars et al., 2014; Jenkins et al., 2015) social functioning, occupational stability, living standard, wellbeing, physical health (Bellis et al., 2014; Holman et al., 2016) and risk of premature death (Brown et al., 2009). However, literature reviews highlight a lack of consistency and clarity concerning the definition, measurement and assessment of ACEs 
(McLaughlin, 2016). It is not always clear where the line is drawn between normative stress experiences and ACEs. There is also ambiguity as to whether low socioeconomic status (SES) should be considered a form of ACE, or an independent factor contributing to negative adult outcomes. Risk of exposure to ACEs may be more common in low SES environments (Hatch and Dohrenwend, 2007; Soares et al., 2016). Poverty is a powerful predictor of mental illness, and it predicts many other causes of mental distress (Read, 2010).

There are links between poverty, brain development and behaviour that suggest that children with low SES have a higher chance of behaving in ways that could harm their health, and reduce life expectancy. Evidence is emerging that our capacity to resist environments that tempt us to overeat, smoke, drink excessively, or be physically inactive is influenced by the strength of our 'executive functioning'. Executive functioning skills (EFs) refer to the mental processes required when you have to pay attention, when going on 'auto pilot' would be illadvised or insufficient. EFs are essential for mental health, physical health, and success; and for cognitive, social and psychological development (Diamond, 2013). Children living in low SES settings potentially face a double hit: living in environments that contain more cues for unhealthy behaviours, combined with exposure to psychosocial environments which may increase vulnerability to a reduction in the EFs to resist those cues (Stringhini et al., 2010; Moffitt et al., 2011). Further research is required to examine the roles of emotional processing and EFs in linking ACEs with negative outcomes in adulthood (McLaughlin, 2016).

There are several different methods of measuring ACEs. Most require self or parent report and assess up to twenty factors, most often including: parental incarceration, domestic violence, household mental illness, familial suicide and household alcohol or substance abuse. The methods usually use numeric, cumulative risk scoring methodology (Bethell et al., 
ACEs: Evidence, Gaps, Evaluation and Future Priorities

2017a). The same review suggests that research into a single standardised ACE measuring method would help accurately evaluate the link between ACEs and long-term outcomes.

Many studies in this area of research are relatively small, not population based and retrospective, which presents challenges for the evidence base as they are difficult to validate. Adults' recollection of childhood experiences can be biased by their subsequent health and wellbeing (Reuben et al., 2016). Prospective studies provide the strongest data but the number of such studies is limited and fixed by the specific cultural and social context of the period in which they were carried out. Further research is required to address aspects of this field, such as whether particular sensitive periods exist for first exposure to ACEs during childhood. Some studies suggest that early-childhood exposure increases risk of negative adult outcomes including increased susceptibility to mental health problems (Sheridan et al., 2012), other studies suggest that the time of first exposure has no influence on particular outcomes, such as suicide risk (Gomez et al., 2017). It is still unclear how protective and resilience factors influence whether an individual will develop negative outcomes in adulthood. Little information exists that allows us to predict which children will do well and remain resilient and which will develop negative outcomes after being exposed to ACEs. These gaps limit the ability to decide the best ways to encourage development of resilience in exposed children, or whether it would be better to strengthen their capacity to cope with stressors.

The biological pathways and the developmental mechanisms involved linking ACEs to adult outcomes remain poorly understood. The degree to which negative outcomes are mediated through either continued adversity, or through the ACE being embedded within behavioural, neuropsychological, immune, neuroendocrine or epigenetic change needs to be determined. Mechanisms which are being investigated and requiring further research include epigenetic changes (Vaiserman, 2015), neurobiological effects (McCrory et al., 2017) and 
ACEs: Evidence, Gaps, Evaluation and Future Priorities

other biological correlates (Danese and McEwen, 2012), including changes in the biological stress response (Kalmakis and Chandler, 2015).

Physical health outcomes warrant further research to understand the mechanisms linking them with ACEs; these include cancer (Holman et al., 2016), diabetes (Huang et al., 2015; Huffhines et al., 2016), chronic pain (Nelson et al., 2017) and post-traumatic growth phenomena (Sapienza and Masten, 2011). The mechanisms underlying poor mental health and wellbeing following ACEs need further investigation, including substance abuse vulnerability (Somaini et al., 2011), suicide (Sachs-Ericsson et al., 2016), intimate partner violence (Montalvo-Liendo et al., 2015), psychosis (Dvir et al., 2013), and homelessness (Davies and Allen, 2017).

Screening that can identify children affected by ACEs needs additional research (Finkelhor, 2017), in conjunction with an improved understanding of the accuracy of ACE self-report, which can entail a risk of false-negative reports (Hardt and Rutter, 2004; Anda et al., 2010). Many studies are not population-based, making studies small and potentially biased. There are few studies which address ACE risk in particular groups (for example, BME communities or high-risk subgroups such as multiply deprived children living with serious parental mental illnesses). There is also a lack of studies evaluating the outcomes of ACE exposure in developing countries (Sapienza and Masten, 2011).

It is likely that different types of ACE create risk for adulthood in varying ways but there is a lack of clarity in this area. Literature has highlighted this problem and prioritises future research that can tease out the effects of particular types of ACEs on different outcomes (Humphreys and Zeanah, 2015; McLaughlin, 2016). For example, one systematic review reported associations between physical abuse and psychological abuse and any type of cancer, and an association between sexual abuse with specific types of cancer. However, the same review also identified two studies that reported no association between physical and 
ACEs: Evidence, Gaps, Evaluation and Future Priorities

sexual abuse and cancer (Holman et al., 2016). Another systematic review comparing the relationship between different types of ACEs and diabetes reported that parental neglect had the strongest influence, while physical abuse had the weakest influence on later risk of developing diabetes (Huang et al., 2015).

Long-term adversity appears to be more damaging than single events. Many children are not exposed to just one type of ACE - adversity is often experienced in the context of a so-called broader 'risky environment' (Cicchetti and Toth, 2005). For example, poverty and parental substance dependence are linked to child deprivation, neglect and a lack of exposure to resilience factors. Chronic exposure to ACEs predicts the greatest negative outcomes in general (Anda et al., 2010; Danese and McEwen, 2012) with an increased risk for multiple negative outcomes including for psychosis (Dvir et al., 2013) and asthma (Exley et al., 2015). Different people react differently to the same ACEs, leading to a range of outcomes in exposed individuals. Further research is required to understand the reasons for individual differences following ACE exposure, including but not limited to research into resilience and vulnerability (McLaughlin, 2016; Traub and Boynton-Jarrett, 2017) as well as the effects of culture (Sapienza and Masten, 2011), ethnicity and gender (Kajeepeta et al., 2015), and mediating factors (Kalmakis and Chandler, 2015) including cognitive risk factors (Liu et al., 2017).

\section{Quality of the evidence-base for early-years interventions}

A review examining the evidence base for youth interventions lists twenty-seven broadly psychotherapeutic programmes that are relevant and recognised as 'well established' or ‘probably efficacious' and can be considered as evidence-based psychotherapies (Weisz et al., 2015). Overall, there is a lack of evidence-based interventions (Bryson et al., 2017), and few for certain populations. Many population groups need particular attention, including 
ACEs: Evidence, Gaps, Evaluation and Future Priorities

children in foster care (Hambrick et al., 2016), children at risk of suicide (Sachs-Ericsson et al., 2016), those experiencing intimate partner violence in the family (Montalvo-Liendo et al., 2015), children of obese women (McDonnell and Garbers, 2017), ethnic minorities (Burnette and Figley, 2017), children with co-morbid psychosis (Dvir et al., 2013), and young people experiencing homelessness (Davies and Allen, 2017).

It has also been suggested that we need to develop different interventions for different settings, such as those focused on families (Figley and Burnette, 2017), those with a whole community focus (Bethell et al., 2017b) and interventions that may be used in primary care (Bransford and Blizard, 2016) as well as in psychiatric and residential treatment services (Bryson et al., 2017). Further evidence needs to be gathered on how to engage families in interventions and the value of doing so (Bethell et al., 2017b). We need to understand the barriers to implementing trauma informed care (TIC) and how sustainable changes in practice are following TIC training (Wilson et al., 2017). TIC is a concept developed in the US to address the fact that many people in contact with mental health services have experienced trauma and to avoid staff practices in mental healthcare re-traumatising these people: for example, by pressuring a patient to accept medication which mimics previous experiences of powerlessness (Sweeney et al., 2016). Finally, the feedback and perception of those who use mental health services and interventions need to be collected to better understand any issues regarding the mental health service experience and existing barriers to asking about childhood abuse (Read et al., 2018).

Literature reviews suggest that the outcomes of interventions need better evaluation (Bethell et al., 2017b). To be able to apply interventions effectively, further research, development and evaluation of particular approaches and interventions is necessary for ACE screening tools/approaches (Finkelhor, 2017), preventative interventions (Mayer and Thursby, 2012; Larkin et al., 2014), trauma informed educational approaches (Brunzell et al., 
ACEs: Evidence, Gaps, Evaluation and Future Priorities

2016; Wiest-Stevenson and Lee, 2016) and TIC approaches (Oral et al., 2016; Bryson et al., 2017). Reviews highlight the need for more information on the overall costs of assessing and addressing ACEs (Bethell et al., 2017b), cost-benefit analyses (Finkelhor, 2017), measures of costs saved (Mayer and Thursby, 2012) and cost-effectiveness (Larkin et al., 2012). Research shows that the evidence base for many parent/family interventions aimed at improving parenting ability and child outcomes is relatively poor and therefore suggests that interventions must be appropriately targeted for optimal effect (Juffer et al., 2005). In particular it is increasingly understood that the most vulnerable or most at risk children are also the most sensitive to intervention. Intervention personalisation, where interventions are tailored to the individual child, the individual family and age or developmental stage, may be important and necessary. Not all interventions are good for all children. More understanding of the optimal timing of an intervention is needed, in terms of how the intervention interacts with child experiences and characteristics (Hambrick et al., 2016), and possible sensitive periods during development (Huang et al., 2015; McCrory et al., 2017).

\section{Local and national government policies for early-years interventions}

It is claimed that evidence-based intervention programmes are little used in practice (Weisz et al., 2015). Some practitioners may prefer nondirective styles of intervention that are not evidence-based but rather have ideological backing. The programmes can appear too tightly organised and lead to a lack of motivation for the young patient or the therapist. There is also a lack of dissemination of new and developing interventions to those commissioning or delivering services. One 2016 review of TIC approaches mentioned the US as the only nation to have a national policy related to trauma (Sweeney et al., 2016). The same review describes TIC approaches as only beginning to reach the UK and having had little impact in the UK so far, even though there is evidence that TIC systems are effective and can benefit both staff 
ACEs: Evidence, Gaps, Evaluation and Future Priorities

and those receiving mental healthcare, at least in the short term. ACEs and trauma awareness were included in Scotland's Mental Health Strategy (2017-2027) (Scottish Government, 2017) and the Scottish government commissioned NHS Education for Scotland to develop a National Trauma Skills and Knowledge Framework and a National Training Plan for practitioners. Strategy documents on gender sensitive services that included trauma awareness were published by the Department of Health (Golding and Duggal, 2011). The Department of Health also made recommendations in 2003 that made enquiry about abuse compulsory in mental health settings, and initiated a programme aimed at training staff (Department of Health, 2003). However, there is little evidence that trauma enquiry occurs in routine practice. Updates to the National Institute for Health and Clinical Excellence guidelines, such as to the guidance for the management of schizophrenia (NICE, 2014), may help prompt TIC being adopted. One review lists a number of barriers to implementation of TIC, including resistance by practitioners to the causal link between trauma and ACE to later risk of psychosis and mental distress, and the fact that continuous change to UK public services leads many to be wary of new initiatives (NICE, 2014).

Poor dissemination and a lack of accessibility and visibility of research represent challenges to the implementation of new evidence-based practice. One review of the assessment and response to ACEs highlighted a lack of awareness of new research and care strategies by nursing staff, who form the largest proportion of frontline healthcare staff (Waite et al., 2010).

\section{Future priorities}

The UK Research Councils recognise that this research into ACEs and their implications requires additional funding. This is particularly true for mental health; several funding bodies are highlighting the relevance of early life experience (e.g. 2017 Medical Research Council 
mental health strategy, Medical Research Council, 2017). Funding mechanisms are designed to create competition between expert groups and universities rather than collaboration where groups with common or overlapping expertise are drawn together. Public and third sector funding bodies put out researcher-led or themed calls - however, there may be little input from stakeholders or affected groups. There is a corresponding limitation in the effective setting of research priorities on a national basis. The James Lind Alliance (JLA) is an example of a mechanism that allows 'Priority Setting Partnerships' to be established with the aim of bringing patients, carers and clinicians together to identify research priorities (www.jla.nihr.ac.uk/jla-guidebook/chapter-3/prioritysetting-partnerships.htm). There is need for better coordination between research about ACEs and associated outcomes including mechanisms and research on the effectiveness of interventions, which is separately funded. Much of the scientific research in this field is fragmented and focused on specific health or social outcomes, without being more widely framed. There is an urgent need for rigorous reviews of the evidence concerning ACEs. NICE produces authoritative reviews on interventions for individual disorders but is limited by its restriction to health and social care. This area was partly the key remit of the National Academy of Parenting Practitioners (NAPP) (Department for Education, 2012), which closed in 2010. The NAPP aimed to provide an understanding for commissioners and educators of the quality of the evidence base for the range of parenting interventions, monitor their use and co-ordinate the development of research projects and measures. The potential value of such a body remains. The model could be extended to include educational and primary care interventions. More work is needed to improve information sharing about children's exposures to ACEs, as well as sharing and accessibility of evidence. NAPP recommended creating a 'living' evidence synthesis and dissemination mechanism to use existing dissemination platforms to ensure that information reaches those involved in children's health services; using existing rapid-cycle learning 
ACEs: Evidence, Gaps, Evaluation and Future Priorities

platforms to maintain networks of families and professionals to promote cross-sector learning and engagement; and developing open source training and tools (Bethell et al., 2017b).

As highlighted above, there is a need for improved coordination of research in this area in order to ensure children and young people living with adversity are at the heart of the process to help set priorities and programmes whereby evidence gaps are can be addressed. One mechanism may involve an expert commission able to develop a portfolio of research agreed by a representative panel of experts by experience alongside researchers crossing disciplinary boundaries, and setting out a series of agreed aims, priorities, milestones and outputs.

\section{Acknowledgements}

This article is based on the evidence submitted by the Academy of Medical Sciences to the House of Commons' Science and Technology Committee inquiry into evidence-based earlyyears intervention (e-mail: info@acmedsci.ac.uk). The article includes contributions from Eric Taylor (Institute of Psychiatry, Psychology and Neuroscience, King's College London) and David Gunnell (Population Health Sciences, University of Bristol).

\section{References}

Anda, R. F., Butchart, A., Fellitti, V. J. and Brown, D. W. (2010) Building a framework for global surveillance of the public health implications of adverse childhood experiences. American Journal of Preventive Medicine, 39, 1, 93-8.

Bellis, M. A., Hughes, K., Leckenby, N., Hardcastle, K. A., Perkins, C. and Lowey, H. (2014) Measuring mortality and the burden of adult disease associated with adverse childhood experiences in England: a national survey. Journal of Public Health, 37, 3, $445-54$. 
ACEs: Evidence, Gaps, Evaluation and Future Priorities

Bethell, C. D., Carle, A., Hudziak, J., Gombojav, N., Powers, K., Wade, R. and Braveman, P. (2017a) Methods to assess adverse childhood experiences of children and families: toward approaches to promote child well-being in policy and practice. Academic Pediatrics, 17, 7S, S51-69.

Bethell, C. D., Solloway, M. R., Guinosso, S., Hassink, S., Srivastav, A., Ford, D. and Simpson, L. A. (2017b) Prioritising possibilities for child and family health: an agenda to address adverse childhood experiences and foster the social and emotional roots of well-being in paediatrics. Academic Pediatrics, 17, 7S, S36-50.

Bransford, C. L. and Blizard, R. A. (2016) Viewing psychopathology through a trauma lens. Social Work Mental Health, 15, 1, 80-98.

Brown, D. W., Anda, R. F., Tiemeier, H., Felitti, V. J., Edwards, V. J., Croft, J. B. and Giles, W. H. (2009) Adverse childhood experiences and the risk of premature mortality. American Journal of Preventive Medicine, 37, 5, 389-96.

Brunzell, T., Stokes, H. and Waters, L. (2016) Trauma-informed positive education: using positive psychology to strengthen vulnerable students. Contemporary School of Psychology, 20, 1, 63-83.

Bryson, S. A., Gauvin, E., Jamieson, A., Rathgeber, M., Faulkner-Gibson, L., Bell, S., Davidson, J., Russel, J. and Burke, S. (2017) What are the strategies for implementing trauma-informed care in youth inpatient psychiatric and residential treatment settings? A realist systematic review. International Journal of Mental Health Systems, 11, 36, doi: 10.1186/s13033-017-0137-3.

Burnette, C. E. and Figley, C. R. (2017) Historical oppression, resilience and transcendence: can a holistic framework help explain violence experiences by indigenous people? Social Work, 62, 1, 37-44. 
ACEs: Evidence, Gaps, Evaluation and Future Priorities

Cicchetti, D. and Toth, S. L. (2005) Child maltreatment. Annual Review of Clinical Psychology, 1, 409-38, doi: 10.1146/annurev.clinpsy.1.102803.144029.

Danese, A. and McEwen, B. S. (2012) Adverse childhood experiences, allostasis, allostatic load, and age-related disease. Physiology and Behavior, 106, 1, 29-39.

Davies, B. R. and Allen, N. B. (2017) Trauma and homelessness in youth: psychopathology and intervention. Clinical Psychology Review, 54, 17-28.

Department for Education (2012) Evaluation of the NAPP's Training Offer in Evidence Based Parenting Programmes. https://www.gov.uk/government/uploads/system/ uploads/attachment_data/file/183457/DFE-RR186.pdf [accessed 13.02.2019].

Department of Health (2003) Women's Mental Health, Into the Mainstream: Implementation Guidance. London: Department of Health.

Diamond, A. (2013) Executive functions, Annual Review of Psychology, 64, 135-68.

Dvir, Y., Denietolis, B. and Frazier, J. A. (2013) Childhood trauma and psychosis. Child and Adolescent Psychiatric Clinics of North America, 22, 4, 629-41.

Exley, D., Norman, A. and Hyland, M. (2015) Adverse childhood experience and asthma onset: a systematic review. European Respiratory Review, 24, 136, 299-305.

Figley, C. R. and Burnette, C. E. (2017) Building bridges: connecting systemic trauma and family resilience in the study and treatment of diverse traumatized families. Traumatology, 23, 1, 95-101.

Finkelhor, D. (2017) Screening for adverse childhood experiences (ACEs): cautions and suggestions. Child Abuse and Neglect, 85, 174-9.

Geoffroy, M-C., Gunnell, D. and Power, C. (2014) Prenatal and childhood antecedents of suicide: 50-year follow-up of the 1958 British Birth Cohort Study. Psychological Medicine, 44, 1245-56. 
ACEs: Evidence, Gaps, Evaluation and Future Priorities

Golding, N. and Duggal, A. (2011) Commissioning Services for Women and Children Who Have Experienced Violence or Abuse - A Guide for Health Commissioners. London: Department of Health.

Gomez, S. H., Tse, J., Wang, Y., Turner, B., Millner, A. J., Nock, M.K. and Dunn, E. C. (2017) Are there sensitive periods when child maltreatment substantially elevates suicide risk? Results from a nationally representative sample of adolescents. Depression and Anxiety, 34, 8, 734-41.

Hambrick, E. P., Oppenheim-Weller, S., N’zi, A. M. and Taussig, H. N. (2016) Mental health interventions for children in foster care: a systematic review. Children and Youth Service Review, 70, 65-77.

Hardt, J. and Rutter, M. (2004) Validity of adult retrospective reports of adverse childhood experiences: review of the evidence. Journal of Child Psychology and Psychiatry, 45, $2,260-73$.

Hatch, S. and Dohrenwend, B. (2007) Distribution of traumatic and other stressful life events by race/ ethnicity, gender, SES and age: a review of the research. American Journal of Community Psychology, 40, 3-4, 313-32.

Holman, D. M., Ports, K. A., Buchanan, N. D., Hawkins, N. A., Merrick, M. T., Metzler, M. and Trivers, K. F. (2016) The association between adverse childhood experiences and risk of cancer in adulthood: a systematic review of the literature. Pediatrics, 138, S1, S81-91.

Huang, H., Yan, P., Shan, Z., Chen, S., Li, M., Luo, C., Gao, H., Hao, L. and Liu, L. (2015) Adverse childhood experiences and risk of type 2 diabetes: a systematic review and meta-analysis. Metabolism, 64, 11, 1408-18. 
ACEs: Evidence, Gaps, Evaluation and Future Priorities

Huffhines, L., Noser, A. and Patton, S. R. (2016) The link between adverse childhood experiences and diabetes. Current Diabetes Reports, 16, 6, 54, doi: 10.1007/s11892016-0740-8.

Humphreys, K. L. and Zeanah, C. H. (2015) Deviations from the expectable environment in early childhood and emerging psychopathology. Neuropsychopharmacology, 40, 1, $154-70$.

Juffer, F., Bakermans-Kranenburg, M. J. and van IJzendoorn, M. H. (2005) The importance of parenting in the development of disorganized attachment: evidence from a preventive intervention study in adoptive families. Journal of Child Psychology and Psychiatry, 46, 3, 263-74.

Jenkins, J., Madigan, S. and Arseneault, L. (2015) Psychosocial adversity. In M. Rutter, D. Bishop, D. Pine, S. Scott, J. S. Stevenson, E. Taylor, and A. Thapar (eds.), Rutter's Child and Adolescent Psychiatry, Oxford: Wiley-Blackwell, 330.

Kalmakis, K. A. and Chandler, G. E. (2015) Health consequences of adverse childhood experiences: a systematic review. Journal of the American Association of Nurse Practitioners, 27, 8, 457-65.

Kajeepeta, S., Gelaye, B., Jackson, C. L. and Williams, M. A. (2015) Adverse childhood experiences are associated with adult sleep disorders: a systematic review. Sleep Medicine, 16, 3, 320-30.

Larkin, H., Shields, J. J. and Anda, R. F. (2012) The health and social consequences of adverse childhood experiences across the lifespan: an introduction to prevention and intervention in the community. Journal of Preventive Intervention in the Community, 40, 4, 263-70. 
ACEs: Evidence, Gaps, Evaluation and Future Priorities

Larkin, H., Felitti, V. J. and Anda, R. F. (2014) Social work and adverse childhood experiences research: implications for practice and health policy. Social Work and Public Health, 29, 1, 1-16.

Lereya, S. T., Winsper, C., Heron, H., Lewis, G., Gunnell, D., Fisher, H. L. and Wolke, D. (2013) Being bullied during childhood and the prospective pathways to self-harm in late adolescence. Journal of the American Academy of Child and Adolescent Psychiatry, 52, 608-18.

Liu, H., Atrooz, F., Salvi, A. and Salim, S. (2017) Behavioural and cognitive impact of early life stress: insights from an animal model. Progress in Neuropsychopharmacology and Biological Psychiatry, 78, 88-9.

Mars, B., Heron, J., Crane, C., Hawton, K., Kidger, J., Lewis, G., Macleod, J., Tilling, K. and Gunnell, D. (2014) Differences in risk factors for self-harm with and without suicidal intent: findings from the ALSPAC cohort. Journal of Affective Disorders, 168, 40714.

Mayer, L. M. and Thursby, E. (2012) Adolescent parents and their children: a multifaceted approach to prevention of adverse childhood experiences. Journal of Preventive Intervention in the Community, 40, 4, 304-12.

McCrory, E. J., Gerin, M. I. and Viding, E. (2017) Annual research review: childhood maltreatment, latent vulnerability and the shift to preventative psychiatry - the contribution of functional brain imaging. Journal of Child Psychology and Psychiatry, $58,4,338-57$.

McDonnell, C. J. and Garbers, S. V. (2017) Adverse childhood experiences and obesity: systematic review of behavioural interventions for women. Psychological Trauma, 16, $5,1-195$. 
ACEs: Evidence, Gaps, Evaluation and Future Priorities

McLaughlin, K. A. (2016) Future directions in childhood adversity and youth psychology. Journal of Clinical Child Adolescent Psychology, 45, 3, 361-82.

Medical Research Council (2017) Strategy for Lifelong Mental Health Research.

www.mrc.ac.uk/ documents/pdf/strategy-for-lifelong-mental-health-research/ [accessed 13.02.2019].

Moffitt, T. E., Arseneault, L., Belsky, D., Dickson, N., Hancox, R. J., Harrington, H., Houts, R., Poulton, R., Roberts, B. W., Ross, S., Sears, M. R., Thomson, W. M. and Caspi, A. (2011) A gradient of childhood self-control predicts health, wealth and public safety. Proceedings of the National Academy of Sciences USA, 108, 7, 2693-8.

Montalvo-Liendo, N., Fredland, N., McFarlane, J., Lui, F., Koci, A. F. and Nava, A. (2015) The intersection of partner violence and adverse childhood experiences: implications for research and clinical practice. Issues in Mental Health Nursing, 36, 12, 989-1006.

Nelson, S. M., Cunningham, N. R. and Kashikar-Zuck, S. (2017) A conceptual framework for understanding the role of adverse childhood experiences in paediatric chronic pain. Clinical Journal of Pain, 33, 3, 264-70.

National Institute for Health and Clinical Excellence (NICE) (2014) Psychosis and Schizophrenia in Adults: Prevention and Management. https://www.nice.org.uk/guidance/cg178 [accessed 13.02.2019].

Oral, R., Ramirez, M., Coohey, C., Nakada, S., Walz, A., Kuntz, A., Benoit, J. and Peek-Asa, C. (2016) Adverse childhood experiences and trauma informed care: the future of health care. Pediatric Research, 79, 1-2, 227-33.

Read, J. (2010) Can poverty drive you mad? 'Schizophrenia', socio-economic status and the case for primary prevention. New Zealand Journal of Psychology, 39, 2, 7-19. 
ACEs: Evidence, Gaps, Evaluation and Future Priorities

Read, J., Harper, D., Tucker, I. and Kennedy, A. (2018) Do adult mental health services identify child abuse and neglect? A systematic review. International Journal of Mental Health Nursing, 27, 1, 7-19.

Reuben, A., Moffitt, T. E., Caspi, A., Belsky, D. W., Harrington, H., Schroeder, F., Hogan, S., Ramrakha, S., Poulton, R. and Danese, A. (2016) Lest we forget: comparing retrospective and prospective assessments of adverse childhood experiences in the prediction of adult health. Child Psychology and Psychiatry, 57, 10, 1103-12.

Sachs-Ericsson, N. J., Rushing, N. C., Stanley, I. H. and Sheffler, J. (2016) In my end is my beginning: developmental trajectories of adverse childhood experiences to late-life suicide. Aging and Mental Health, 20, 2, 139-65.

Sapienza, J. K. and Masten, A. S. (2011) Understanding and promoting resilience in children and youth. Current Opinion in Psychiatry, 24, 4, 267-73.

Scottish Government (2017) Scotland's Mental Health Strategy (2017-2027). http://www.gov.scot/Resource/0051/00516047.pdf [accessed 13.02.2019].

Sheridan, M. A., Fox, N. A., Zeanah, C. H., McLaughlin, K. A. and Nelson, C. A. (2012) Variation in neural development as a result of exposure to institutionalization early in childhood. Proceedings of the National Academy of Sciences USA, 109, 12927-32.

Soares, A. L., Howe, L. D., Matijasevich, A., Wehrmeister, F. C., Menezes, A. M. and Gonçalves, H. (2016) Adverse childhood experiences: prevalence and related factors in adolescents of a Brazilian birth cohort. Child Abuse and Neglect, 51, 21-30.

Somaini, L., Donnini, C., Manfredini, M., Raggi, M. A., Saracino, M. A., Gerra, M. L., Amore, M., Leonardi, C., Serpelloni, G. and Gerra, G. (2011) Adverse childhood experiences, genetic polymorphisms and neurochemical correlates in experimentation with psychotropic drugs among adolescents. Neuroscience and Biobehavioral Reviews, 35, 8, 1771-8. 
ACEs: Evidence, Gaps, Evaluation and Future Priorities

Stringhini, S., Sabia, S., Shipley, M., Brunner, E., Nabi, H., Kivimaki, M. and SinghManoux, A. (2010) Association of socioeconomic position with health behaviors and mortality. JAMA, 303, 12, 1159-66.

Sweeney, A., Clement, S., Filson, B. and Kennedy, A. (2016) Trauma-informed mental healthcare in the UK: what is it and how can we further its development? Mental Health Review Journal, 21, 3, 174-92.

Traub, F. and Boynton-Jarrett, R. (2017) Modifiable resilience factors to childhood adversity for clinical pediatric practice. Pediatrics, 139, 5, e20162569, doi: 10.1542/peds.20162569.

Vaiserman, A. M. (2015) Epigenetic programming by early-life stress: evidence from human populations. Developmental Dynamics, 244, 3, 254-65.

Waite, R., Gerrity, P. and Arango, R. (2010) Assessment for and response to adverse childhood experiences. Journal of Psychosocial Nursing and Mental Health Services, $48,12,51-61$.

Wiest-Stevenson, C. and Lee, C. (2016) Trauma-informed schools, Journal of Evidence Informed Social Work, 13, 5, 498-503.

Weisz, J. R., Ng, M. Y. and Lau, N. (2015) Psychological interventions: overview and critical issues for the field. In M. Rutter, D. Bishop, D. Pine, S. Scott, J. S. Stevenson, E. Taylor, and A. Thapar (eds.), Rutter's Child and Adolescent Psychiatry, Oxford: Wiley-Blackwell, 461-82.

Wilson, A., Hutchinson, M. and Hurley, J. (2017) Literature review of trauma-informed care: implications for mental health nurses working in acute inpatient settings in Australia. International Journal of Mental Health Nursing, 26, 4, 326-43. 